\title{
UTILITY OF ANTIGEN DETECTION TEST AND POLYMERASE CHAIN REACTION IN THE DIFFERENTIATION OF TUBERCULOUS AND NON-TUBERCULOUS MYCOBACTERIA
}

\author{
YOGITA SINGH, RAJI VASANTH, SHRIKALA BALIGA, BIRANTHABAIL DHANASHREE* \\ Department of Microbiology, Kasturba Medical College, Manipal University, Mangalore, Karnataka, India. Email: dbiranthabail@yahoo.co.in \\ Received: 03 March 2017, Revised and Accepted: 24 March 2017
}

ABSTRACT

Objectives: Cultivation and identification of mycobacteria to species level remains difficult and time-consuming. Hence, easy and rapid diagnostic methods are necessary for the differentiation of Mycobacterium tuberculosis (MTB) from non-tuberculous mycobacteria (NTM). The present study aims to detect and differentiate MTB from NTM isolated from clinical samples by immunochromatographic test (ICT) and polymerase chain reaction (PCR).

Methods: Over a period of 1 year, clinical samples $(n=496)$ received from suspected cases of TB, at the Department of Microbiology, Kasturba Medical College Hospital, Mangalore were cultured to isolate Mycobacterium spp. Identification of all the isolates was done by conventional biochemical technique, ICT, and PCR.

Results: Among the 496 samples processed, 49 (9.87\%) were acid-fast bacilli smear positive and 59 (11.89\%) samples showed the growth of Mycobacterium spp. Among these, 10 were rapid growers, 49 were slow-growing mycobacteria, out of which 30 were MTB as identified by conventional biochemical reaction. Out of 59 Mycobacterial isolates subjected to ICT for the detection of MPT 64 antigen, only 28 were identified as MTB. However, all the 30 isolates were correctly identified as MTB by PCR.

Conclusion: Hence, PCR is essential for rapid differentiation of non-tuberculous Mycobacterium from MTB. False negative results seen with immunochromatographic MPT 64 antigen assay could be due to mutations within the mpt64 gene. Further studies are necessary to characterize these PCR-positive and immunochromatographic assay negative MTB isolates.

Keywords: Mycobacterium tuberculosis, Polymerase chain reaction, Culture, Antigen detection.

(c) 2017 The Authors. Published by Innovare Academic Sciences Pvt Ltd. This is an open access article under the CC BY license (http://creativecommons. org/licenses/by/4. 0/) DOI: http://dx.doi.org/10.22159/ajpcr.2017.v10i6.18261

\section{INTRODUCTION}

Tuberculosis (TB) is one of the major public health problems worldwide and India accounts for the highest TB burden in the world, accounting for 2.2 million new TB cases each year [1]. Drug resistance in Mycobacterium tuberculosis (MTB) is a growing concern. As per the Global TB report 2014 data, there were 35,385 laboratory confirmed rifampicin resistant/MDR TB cases in India alone [2]. Hence, the important challenges before microbiologists are early and accurate diagnosis and differentiation of infection caused by MTB from that of atypical Mycobacteria. Rapid and accurate differentiation of MTB from non-tuberculous mycobacteria (NTM) will help in early initiation of appropriate treatment which in turn will stop the emergence and spread of multidrug-resistant TB and extensively drug-resistant TB, and also reduce morbidity and mortality.

Conventional methods for the diagnosis of TB such as microscopy, though rapid, lack sensitivity. Culture of mycobacteria and drug susceptibility testing by conventional method is time-consuming (approximately 8-10 weeks). Identification of mycobacterial growth to species level by conventional method delays the reports further by 2 more weeks [3]. Rapid and accurate differentiation of NTM from MTB is also necessary to avoid inappropriate treatment, thereby minimizes the emergence and spread of drug-resistant strains. Mycobacterial growth indicator tube (MGIT) is expensive and takes a minimum of 7-10 days for the growth to be indicated. Moreover, MGIT will detect the presence or absence of growth but cannot differentiate MTB from that of NTM [4]. Hence, an attempt is made in the present study to detect and differentiate MTB from that of NTM by rapid immunochromatographic antigen detection test and PCR.

\section{METHODS}

Clinical samples included in the study

Over a period of 1 year (January to December 2014), 461 samples that consisted of bronchial alveolar lavage (62), cerebrospinal fluid (CSF) (73), urine (50), pus (52), and sputum (261), received at the Department of Microbiology, Kasturba Medical College Hospital, Mangalore, from adult patients who were more than 18 years of age, were included in the study by following random sampling method. Individuals who were $<18$ years of age and those adults who were on antibiotic and anti-TB treatment were excluded from the study. This study was approved by the Institutional Ethics Committee of Kasturba Medical College, Mangalore. All samples were transported at room temperature $\left(25-30^{\circ} \mathrm{C}\right)$ and processed within 30 minutes of their receipt.

\section{Microscopic examination of the samples}

Smears were prepared directly from all samples and also from the concentrated deposits of the same samples and stained by Ziehl-Neelsen's staining to demonstrate acid-fast bacilli and interpreted as per the Revised National TB Control Programme guidelines [5].

\section{Culture of samples and identification of isolates}

Sterile samples such as CSF were concentrated by centrifugation and the deposit were inoculated onto the Lowenstein-Jensen (LJ) medium. Samples from non-sterile site such as bronchoalveolar lavage, and pus, sputum were digested/decontaminated by Petroff's method and inoculated onto LJ medium. Cultures were incubated at $37^{\circ} \mathrm{C}$. Cultures were first examined after $72 \mathrm{hrs}$ and later at weekly interval for 8 weeks or until growth occurs. The growth on LJ medium was observed for pigmentation; colony morphology and time taken for growth were 
noted. Smears were prepared from suspected colonies and identified by standard biochemical reactions such as thermostable catalase test, nitrate reduction test, niacin production, and urease test [6].

MPT64 antigen detection by immunochromatographic test (ICT) The SD BIOLINE TB Ag MPT64 RAPID kit was used according to the manufacturer's instruction manual. Three or four colonies of mycobacterial growth from LJ medium were suspended in $200 \mu \mathrm{L}$ of the extraction buffer and $100 \mu \mathrm{L}$ of the suspension was added to the sample well. Incubated for 15 minutes at room temperature. Chromatographic diffusion of a specific MPT64 antigen of MTB complex reacts with anti-MPT64 monoclonal antibodies. Mouse monoclonal anti-MPT64 antibodies were immobilized on a nitrocellulose membrane as the capture material. Another antibody, which recognizes a different epitope of MPT64, has been conjugated with colloidal gold particles, which was used for antigen capture and detection in a sandwich-type assay. The presence of a control band (C) alone indicates a negative result, whereas the presence of two pink color bands one at $\mathrm{C}$ and other at T indicates a positive result. Culture of Mycobacterium H37 RV strain grown on LJ medium was employed as the positive reference control, which gave two pink color bands.

\section{Differentiation of MTB from NTM by polymerase chain reaction} (PCR) [7]

PCR targeting the mycobacterial hsp65 gene was performed on the DNA extracted from colonies of mycobacterial isolates and Mycobacterium $\mathrm{H} 37 \mathrm{RV}$ strain using the primers $\mathrm{F5}^{\prime}$ TGCTCGAGAAGGTCATCGGA-3' and R5'-TCACCTGACCACCGGTGAGA-3' for MTB complex and F5'-GAGGTGGCCAAGAAGACGGAC-3' and R5'AGCAGCGGCTTGCCCTCTGA- $3^{\prime}$ for NTM. PCR cycle included initial denaturation at $95^{\circ} \mathrm{C}$ for 5 minutes, 30 cycles $\left(30\right.$ seconds at $95^{\circ} \mathrm{C}$, 30 seconds at $58^{\circ} \mathrm{C}$ and 60 seconds at $72^{\circ} \mathrm{C}$ ), and a final elongation at $72^{\circ} \mathrm{C}$ for 5 minutes. Amplified product was detected using $2 \%$ agarose gel, stained with ethidium bromide, and visualized under ultraviolet transilluminator. Amplicon size of 195 and 515 bp will indicate the presence of MTB and NTM, respectively.

\section{RESULTS}

Among 59 culture positive samples, $10(16.94 \%)$ were rapid growers and 49 (83.05\%) were slow growers. All rapid growers were biochemically identified as NTM. Among the 49 slow growers, 30 (50.84\%) isolates were MTB as identified by conventional biochemical test. All the biochemically confirmed mycobacterial isolates were subjected to ICT for the detection of TB Ag MPT64 and only 28 (93.33\%) gave the test and control bands and rest of the isolates $(n=31)$ showed only control bands. Hence, only 28 isolates were identified as MTB by ICT. All 59 mycobacterial isolates were subjected to PCR and 30 isolates gave a 195 bp band specific for MTB $h s p 65$ gene and 29 isolates produced 515 bp bands that corresponded to NTM hsp65 gene as shown in Fig. 1. Two MTB isolates tested negative by ICT were positive by PCR and conventional biochemical reactions. Various clinical samples tested and performance of different tests used in the study are shown in Tables 1 and 2.

\section{DISCUSSION}

TB is a public health problem in our country. Detection of TB cases rapidly using a cost-effective method is the need of the hour. Many of the well-equipped microbiology laboratories in the medical colleges of the country still use conventional biochemical reactions for the identification and differentiation of MTB from NTM. This process will delay the initiation of treatment and will lead to the spread of the disease in the community. Hence, many researchers have developed rapid, cost-effective methods such as enzyme-linked immunosorbent assay, PCR and antigen detection test [8]. One such rapid method is the ICT. In this rapid test, MPT64 antigen is detected. Earlier studies have shown that MPT 64 and culture filtrate protein - 2 antigens are found only in MTB complex and are TB-specific candidate antigens [9].

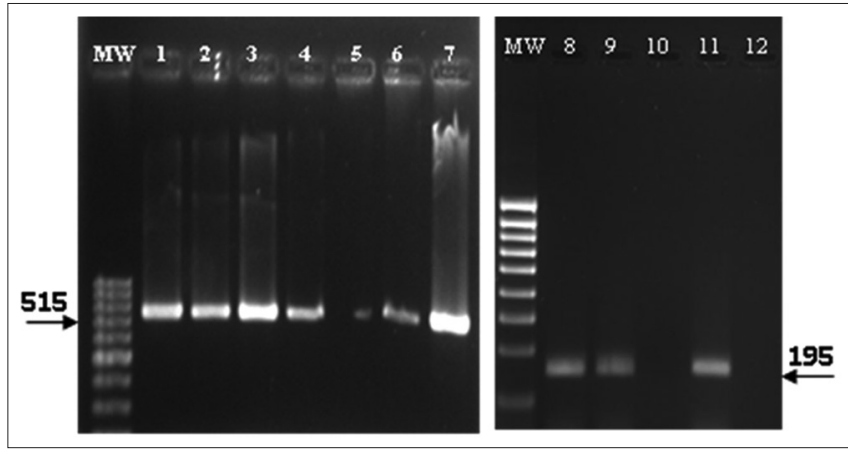

Fig. 1: Agarose gel picture showing the amplification product of polymerase chain reaction (PCR) performed on mycobacterial culture isolates for the detection of hsp65 gene: PCR products of 515 and 195 bp correspond to hsp65 gene of non-tuberculous mycobacteria (NTM) and MTB, respectively. Lanes: Mw: Molecular weight marker; 1, 2, 3, 4, 6: NTM isolates; 5 and 12: Negative control; 7: Positive control (Mycobacterium fortutum); 8, 9: M. tuberculosis complex isolates; 10: Negative culture; 11: Positive control (Mycobacterium H37 RV strain)

Earlier few studies from India and abroad have shown that immunochromatographic TB Ag MPT64 rapid test to be $100 \%$ specific and sensitive [10-13]. However, in the present study, we have found that TB Ag MPT64 rapid test could not identify two isolates of MTB which was otherwise identified as MTB by biochemical test as well as by PCR. Hence, negative results of ICT rapid card tests must be interpreted with caution and such negative results need confirmation by PCR or other alternate tests. In the present study, due to false negative results of ICT, the sensitivity of the test is found to be $93.33 \%$, specificity $100 \%$, positive predictive value to be $100 \%$, and negative predictive value to be $93.5 \%$. However, an earlier Indian study has also found the sensitivity of the test to be $99 \%$ due to one single false negative result. Our culture isolates were only 59, whereas earlier study, they have used 102 mycobacterial isolates from extrapulmonary samples [14]. Hence, further studies are necessary to know whether mycobacterial isolates from different clinical samples behave differently. Another possible explanation for the false negative results could be due to mutations within the mpt64 gene of these strains, which may have led to the production of an incomplete protein as reported by earlier workers $[15,16]$. Hence, DNA sequencing of isolates which tested negative by ICT and positive by PCR are necessary to substantiate the negative findings.

Moreover, ICT test being rapid will markedly reduce the turnaround time in MTB culture and identification. Hence, in spite of small proportion of false negative results, this can be an alternative rapid and cost-effective identification method for the differentiation of MTB from NTM. In the study, PCR was found to be $100 \%$ specific and sensitive when compared with the conventional technique and ICT. PCR though costly is essential for rapid differentiation of NTM from MTC, especially those suspected isolates which are negative by ICT. Thus, PCR can be used as confirmatory test for those isolates which give doubtful result as conventional biochemical tests are time-consuming. In spite of various rapid detection of MTB, control of drug-resistant TB can be brought about only by change in knowledge, attitude, and practice by pharmacist [17].

\section{CONCLUSION}

Detection and differentiation of MTC form NTM by ICT test and/or multiplex PCR will considerably reduce the turnaround time in the laboratory diagnosis of $\mathrm{TB}$, which contributes in a significant way to early treatment and TB control program.

\section{ACKNOWLEDGMENT}

We are grateful to the head of the Department of Microbiology and Dean Kasturba Medical College, Manipal University, for providing permission and necessary facilities to carry out the study. 
Table 1: AFB smear and culture results of the different clinical samples

\begin{tabular}{lllll}
\hline \multirow{2}{*}{ Types of samples (n) } & AFB smear (\%) & & \multicolumn{2}{c}{ Culture (\%) } \\
\cline { 2 - 3 } \cline { 5 - 5 } & Positive & Negative & Positive & Negative \\
\hline BAL (62) & $5(08.06)$ & $57(91.93)$ & $13(20.96)$ & $49(79.03)$ \\
CSF (73) & $1(01.36)$ & $72(98.63)$ & $1(01.36)$ & $72(98.63)$ \\
Urine (50) & $4(08.0)$ & $56(92.0)$ & $5(10.0)$ & $45(90.0)$ \\
Pus (52) & $2(03.84)$ & $50(96.15)$ & $5(09.61)$ & $47(90.38)$ \\
Sputum (261) & $37(14.17)$ & $224(85.82)$ & $35(13.40)$ & $226(86.59)$ \\
Total (496) & $49(9.87)$ & $447(90.12)$ & $59(11.89)$ & $437(88.10)$ \\
\hline
\end{tabular}

BAL: Bronchial alveolar lavage, AFB: Acid-fast bacilli, CSF: Cerebro spinal fluid

Table 2: Results of culture, PCR, and TB Ag MPT64 rapid test

\begin{tabular}{|c|c|c|c|c|c|}
\hline \multirow[t]{2}{*}{ Method } & \multicolumn{2}{|c|}{ MTB complex } & \multicolumn{2}{|c|}{ Non-TB mycobacteria } & \multirow[t]{2}{*}{ Total } \\
\hline & No positive & No negative & No positive & No negative & \\
\hline Conventional method & $30(100 \%)$ & 0 & 29 & 0 & 59 \\
\hline TB Ag MPT64 rapid & $28(93.3 \%)$ & 02 & 0 & 29 & 59 \\
\hline
\end{tabular}

TB: Tuberculosis, PCR: Polymerase chain reaction, MTB: Mycobacterium tuberculosis

\section{REFERENCES}

1. TB India. Revised National Tuberculosis Control Programme. Annual Status Report. Central TB Division, Directorate General of Health Services, Ministry of Health and Family Welfare, Nirman Bhavan, New Delhi; 2014. Available from: http://www.tbcindia.nic.in/index1. php?lang $=1 \&$ level $=1 \&$ sublinkid $=4160 \&$ lid $=2807$. [Last accessed on 2016 Jan 26].

2. Global Tuberculosis Report (2014). Annex 2 Country Profiles. Available from: http://www.who.int/tb/data. [Last accessed on $2015 \mathrm{Jul} 16$ ].

3. Nyamogoba HD, Mbuthia G, Mining S, Kikuvi G, Biegon R, Mpoke S, et al. HIV co-infection with tuberculous and non-tuberculous mycobacteria in Western Kenya: Challenges in the diagnosis and management. Afr Health Sci 2012;12:305-11.

4. Siddiqi SH, Gerdes SR. MGIT ${ }^{\mathrm{TM}}$ Procedure Manual. Mycobacteria Growth Indicator Tube (MGIT). Culture and Drug Susceptibility Demonstration; 2006. p. 15-6.

5. Manual for Sputum Smear Fluorescence Microscopy. Revised National Tuberculosis Control Programme. Central TB Division, Directorate General of Health Services, Ministry of Health and Family Welfare, Nirman Bhavan, New Delhi. Available from: http://www.tbcindia.nic. in/showfile.php?lid=2988. [Last accessed on 2017 Apr 11].

6. Revised National TB Control Programme: Manual of Standard Operating Procedures (SOPs). Culture of Mycobacterium tuberculosis and drug Susceptibility Testing on Solid Medium. Version No. 01.01. New Delhi: Central TB Division, Directorate General of Health Services, Ministry of Health and Family Welfare, Nirman Bhavan; 2009.

7. Kim BJ, Park JH, Lee SA, Kim H, Cha CY, Kook YH, et al. Differentiation of mycobacteria in sputa by duplex polymerase chain reaction for mycobacterial hsp65 gene. Diagn Microbiol Infect Dis 2008;62:193-8.

8. Nath SS. Serological immune diagnosis of tuberculosis using PCR and in-house developed clinical isolate based ELISA. Int J Pharm Pharm Sci 2012;5(3):584-92.

9. Wang RF, Shi C, Lu M, Fang Z, Lu J, Wang F, et al. An improved whole-blood gamma interferon assay based the CFP21-MPT64 fusion protein. Clin Vaccine Immunol 2009;16:686-91.

10. Kumar VG, Urs TA, Ranganath RR. MPT 64 Antigen detection for rapid confirmation of M. tuberculosis isolates. BMC Res Notes 2011;4:79.

11. Shenoy VP, Mukhopadhyay C. Rapid immunochromatographic test for the identification and discrimination of Mycobacterium tuberculosis complex isolates from non-tuberculous mycobacteria. J Clin Diagn Res 2014;8(4):DC13-5

12. Marzouk M, Kahla IB, Hannachi N, Ferjeni A, Salma WB, Ghezal S, et al. Evaluation of an immunochromatographic assay for rapid identification of Mycobacterium tuberculosis complex in clinical isolates. Diagn Microbiol Infect Dis 2011;69(4):396-9.

13. Martin A, Bombeeck D, Mulders W, Fissette K, De Rijk P, Palomino JC. Evaluation of the TB Ag MPT64 rapid test for the identification of Mycobacterium tuberculosis complex. Int J Tuberc Lung Dis 2011;15(5):703-5

14. Maurya AK, Nag VL, Kant S, Kushwaha RA, Kumar M, Mishra V, et al. Evaluation of an immunochromatographic test for discrimination between Mycobacterium tuberculosis complex and non tuberculous mycobacteria in clinical isolates from extra-pulmonary tuberculosis. Indian J Med Res 2012;135(6):901-6.

15. SD Bioline TB Ag MPT64 - Is it TB or Non TB. Available from: Http:// www.alere.es/UserFiles/TBAgMPT64.pdf.

16. Hillemann D, Rüsch-Gerdes S, Richter E. Application of the Capilia TB assay for culture confirmation of Mycobacterium tuberculosis complex isolates. Int J Tuberc Lung Dis 2005;9(12):1409-11.

17. Saranya P, Swathi S, Kousalya K, Praveen D. A prospective interventional study of knowledge, attitude and practice (KAP) towards tuberculosis among patients with Koch's disease. Int J Pharm Pharm Sci 2016;8:58-61. 